Relations industrielles

Industrial Relations

\title{
Equal Pay of Equal Value, A Discussion Paper, Ontario Ministry of Labour, 1976, 106 pp.
}

\section{Jacques Saint-Laurent}

Volume 32, numéro 3, 1977

URI : https://id.erudit.org/iderudit/028812ar

DOI : https://doi.org/10.7202/028812ar

Aller au sommaire du numéro

Éditeur(s)

Département des relations industrielles de l'Université Laval

ISSN

0034-379X (imprimé)

1703-8138 (numérique)

Découvrir la revue

Citer ce compte rendu

Saint-Laurent, J. (1977). Compte rendu de [Equal Pay of Equal Value, $A$ Discussion Paper, Ontario Ministry of Labour, 1976, 106 pp.] Relations industrielles / Industrial Relations, 32(3), 469-470.

https://doi.org/10.7202/028812ar

Tous droits réservés (C) Département des relations industrielles de l'Université Laval, 1977
Ce document est protégé par la loi sur le droit d'auteur. L'utilisation des services d'Érudit (y compris la reproduction) est assujettie à sa politique d'utilisation que vous pouvez consulter en ligne.

https://apropos.erudit.org/fr/usagers/politique-dutilisation/ 
and working conditions in these industries. Although the law has only a limited role in dealing with disputes there, these issues are inherently political and will be treated as such by legislatures. Furthermore, there are no general solutions to labour problems in these areas.

None of these conclusions is particularly noteworthy, and this fact may result from an air of unreality about the conference. The location, a Toronto hotel best known for its artificial waterfall, may have set the tone. All of the academics and most of the government officials in attendance were involved with collective bargaining, so it was hardly surprising that the institution was endorsed. Yet there is ample evidence that the public is at least uneasy about collective bargaining in these sectors. Perhaps the analysis would have been more critical had a few politicians attended. Because three of the papers were on an abstract plane, and the fourth, Matkin's, covered only jurisdiction, few deficiencies in existing models or legislative suggestions were proposed, save that legislation is not very helpful in preventing or ending disputes, and that the private sector is the model to be followed. Again dissident voices might have altered the tone and sharpened the conclusions. On balance, at least one, and perhaps two, of the three objectives were unfulfilled.

Yet the strengths of the book remain. It is a collection of several basic works on the subject, worthy of careful consideration by scholars and practitioners. The Matkin paper is destined to be a standard case study of government policy in labour disputes resolution in Canada. Boivin and Phillips provide a useful introduction to most important issues in the area, and Aaron gives a useful comparative standard against which to judge hopes and performance in Canada. The next step in any examination of collective bargaining in essential and public service sectors is a more detailed analysis of specific problems and issues, e.g., compensation standards in the public sector; bargaining structures; the political role of public sector unions, etc.

Mark THOMPSON

University of British Columbia
Equal Pay for Work of Equal Value. A discussion paper, Ontario Ministry of Labour, 1976, 106 pp.

On est tous habitué à entendre les revendications de ceux qui souhaitent que pour «un travail égal», on reçoive un «salaire égal». Le problème soulevé dans la publication ci-haut mentionnée est légèrement différent ou, tout au moins, formulé de façon plus précise. Il s'agit du problème d'accorder une rémunération égale pour un travail d'une valeur égale.

La première formulation permet de vérifier si, pour un travail semblable, il y a de la discrimination dans la rémunération, par exemple, en fonction du sexe. Mais, lorsque certaines occupations son réservées exclusivement aux femmes, la comparaison n'est plus possible. D'où la nécessité d'introduire le concept de «travail d'une valeur égale». Cela permet la comparaison de la rémunération dans des occupations qui ne sont pas semblables, mais pour lesquelles on peut évaluer la valeur du travail fourni. C'est à ce problème qu'on s'attaque dans cette publication du Ministère du Travail de l'Ontario.

Dans un premier temps, une analyse est faite de la rémunération relative des hommes et des femmes dans des groupes d'occupations de plus en plus spécifiques. Il ressort de cette analyse, basée sur les données du recensement de 1971, que la différence dans la distribution des hommes et des femmes dans les plus importants groupes occupationnels n'explique qu'un sixième de la différence de rémunération entre les uns et les autres. Ce n'est donc pas principalement parce que les femmes appartiennent à des groupes d'occupations différentes qu'elles sont moins bien rémunérées.

Il ressort également de cette analyse que la différence dans la rémunération est d'autant moins considérable que l'on considère des occupations spécifiques à l'intérieur d'un établissement et que les employés de cet établissement sont syndiqués. Enfin, bien que les données sur les différences d'âge d'éducation et d'ancienneté ne soient pas accessibles, il semble bien qu'ils sont responsables d'une partie de la différence dans la rémunération. 
Dans un deuxième temps, une tentative est faite pour élaborer de quelle façon il est possible d'identifier des occupations d'égale valeur. On y discute de la notion de valeur chez les économistes - valeur «objectivement» exprimée sur le marché par le truchement du mécanisme des prix et celle, plus «subjective» qui ressort d'une évaluation des tâches par différentes méthodes. On y souligne également les faiblesses de ces différentes méthodes dans l'établissement de la mesure d'un travail de «valeur égale».

Dans un troisième temps, on souligne quelques implications de l'application du principe «à travail de valeur égale, salaire égal». On y reconnaît la possibilité d'une intervention gouvernementale plus poussée dans le processus de négociation et de détermination des salaires. Cette intervention est-elle désirable? Si oui, sera-t-elle acceptée? De plus, jusqu'à quel point l'application de ce principe ne viendra pas nuire à l'établissement des changements dans les salaires relatifs, nécessaires à l'allocation du travail la plus efficace entre les entreprises, les industries, les occupations, les régions? Et, si des exceptions doivent être faites à cet égard, jusqu'à quel point ne viendront-elles pas miner la confiance dans la possibilité d'appliquer le principe du salaire égal pour un travail de valeur égale? Et quels seront les coûts de l'application d'un tel principe?

Enfin, dans un appendice B, on trouve les législations adoptées par les pays suivants dans le but de rétablir l'équité dans la négociation: la France, les Pays Bas, l'Angleterre, la Nouvelle-Zélande et les Etats-Unis.

Bien sûr, on ne trouvera pas dans cette publication toutes les réponses aux problèmes posés, puisque ce n'était pas là l'intention des auteurs. Mais, on y trouvera suffisamment d'information pour mieux apprécier la complexité de l'application du principe en cause et la difficulté d'en évaluer les coûts.

Jacques SAINT-LAURENT

Université Laval
Behavioral Issues in Management: The Canadian Context by H. C. Jain et R. N. Kanungo, Toronto, McGraw Hill Ryerson Ltd., 1977, 586 pp.

L'accroissement d'une préoccupation renouvelée et élargie pour le facteur humain au sein des organisations, sous l'angle de l'amélioration de la qualité de la vie de travail, a donné naissance à la publication de nombreux ouvrages en sciences du comportement. Ces ouvrages, pour la plupart, sont d'origine américaine; par conséquent, ils font peu référence au contexte canadien. C'est cette lacune que les deux auteurs Jain et Kanungo ont voulu combler en publiant ce recueil de textes et de cas qui traite des aspects les plus importants du comportement des individus au sein des organisations de travail. Pour ce faire, ils ont retenu, pour la dimension théorique surtout, des textes américains largement connus. Sous la dimension empirique et pratique, les auteurs regroupent d'excellents travaux de recherche effectués dans des entreprises canadiennes.

Ce regroupement répond à un cadre théorique emprunté de George Strauss qui tente d'expliciter le jeu des interactions entre les variables suivantes:

- la philosophie de gestion et le choix de croyance

- les processus individuels

- les processus de groupes

- les processus organisationnels

- l'environnement

- les variables résultantes telles que la satisfaction et la performance au niveau des individus et des groupes, enfin l'efficacité organisationnelle

Après avoir présenté leur cadre de référence, les auteurs réussissent des textes qui décrivent dans une première partie de l'ouvrage la place des sciences du comportement dans la gestion des entreprises et plus particulièrement leur impact sur la gestion des ressources hurnaines. La deuxième partie traite de la motivation, des perceptions, des attitudes et du changement individuel. La troisième s'intéresse aux phénomènes de groupe tels que les interactions, le conflit, le pouvoir et le leadership. La dernière traite des relations entre des variables organisationnelles sous l'angle des rapports entre la technologie et la struc- 